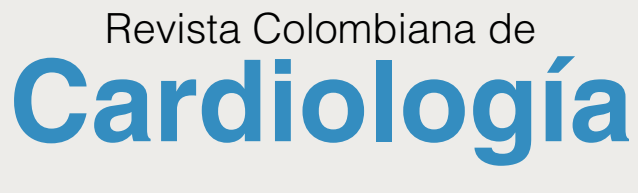

www.elsevier.es/revcolcar

\title{
Utilidad de los catéteres con sensor de fuerza de contacto en la ablación de la fibrilación auricular
}

\section{Usefulness of contact force sensing catheters in atrial fibrillation ablation}

\author{
Antonio Carlos Miranda-Hoyos ${ }^{\mathrm{a}, \mathrm{b}, \mathrm{c}, *}$ y Martín de la Ossa-Aguirre ${ }^{\mathrm{a}, \mathrm{b}, \mathrm{c}}$ \\ a Servicio de Electrofisiología Arritmias y Marcapasos, Clínica San Rafael, Pereira, Colombia \\ b Clínica Comfamiliar Risaralda, Pereira, Colombia \\ c Clínica de la Costa, Barranquilla, Colombia
}

Recibido el 3 de octubre de 2016; aceptado el 6 de octubre de 2016

Disponible en Internet el 9 de noviembre de 2016

\section{Introducción}

La ablación con catéter de radiofrecuencia es hoy en día el estándar de oro en el tratamiento curativo de la mayoría de arritmias, tanto supraventriculares como ventriculares. Con el advenimiento de los sistemas de mapeo tridimensional no fluoroscópico (NavX St. Jude Minneapolis, CARTO Biosense Webster, Rhytmia Boston Scientific) se ha logrado ampliar el rango de utilidad de la misma, mejorando el porcentaje de éxito en procedimientos complejos como la ablación de taquicardia ventricular y la ablación circunferencial de venas pulmonares.

Sin embargo, la transmuralidad de las lesiones, principalmente en la ablación de la fibrilación atrial, siempre ha sido un punto limitante al momento de obtener la menor tasa de recurrencias a corto y largo plazo en los seguimientos clínicos de los pacientes.

Con el objetivo de lograr lesiones transmurales y mejorar el perfil de seguridad se ha desarrollado la tecnología de catéteres de fuerza de contacto para definir la cantidad óptima de fuerza necesaria para lograr líneas de ablación transmurales y mejorar los resultados clínicos.

\footnotetext{
* Autor para correspondencia.

Correo electrónico: fluttermiranda@yahoo.om (A.C. Miranda-Hoyos).
}

Entre los factores que limitan la entrega de energía por radiofrecuencia se encuentran el movimiento constante del corazón, el movimiento respiratorio, el deslizamiento del catéter y la disipación de la energía por el flujo sanguíneo constante. Estudios experimentales in vivo e in vitro han demostrado en forma consecuente que la presión de contacto del electrodo en el tejido es el mayor determinante del diámetro y la profundidad de la lesión ${ }^{1}$.

Esta revisión se propone resaltar las implicaciones del uso de catéteres de ablación de fuerza de contacto en los resultados de la ablación de la fibrilación atrial.

\section{Tecnología del catéter de fuerza de contacto}

En comercio se dispone de dos tipos de plataformas tecnológicas de sensores de fuerza de contacto. El primero utiliza un pequeño resorte con sensores para medir microdeformaciones que conectan la punta del catéter con su eje (Thermocool Smartouch CF, Biosense Webster Inc., Diamond Bar, California, USA) ${ }^{2}$.

El catéter Thermocool Smartouch tiene un pequeño resorte, localizado proximalmente en la punta del electrodo de ablación. El sensor distal es un emisor de señal magnética que está anclado a la punta del electrodo, y proximal a los resortes hay tres sensores magnéticos que miden las microdeflexiones de la punta del catéter. La microdeflexión se calcula determinando la magnitud y el ángulo de la 


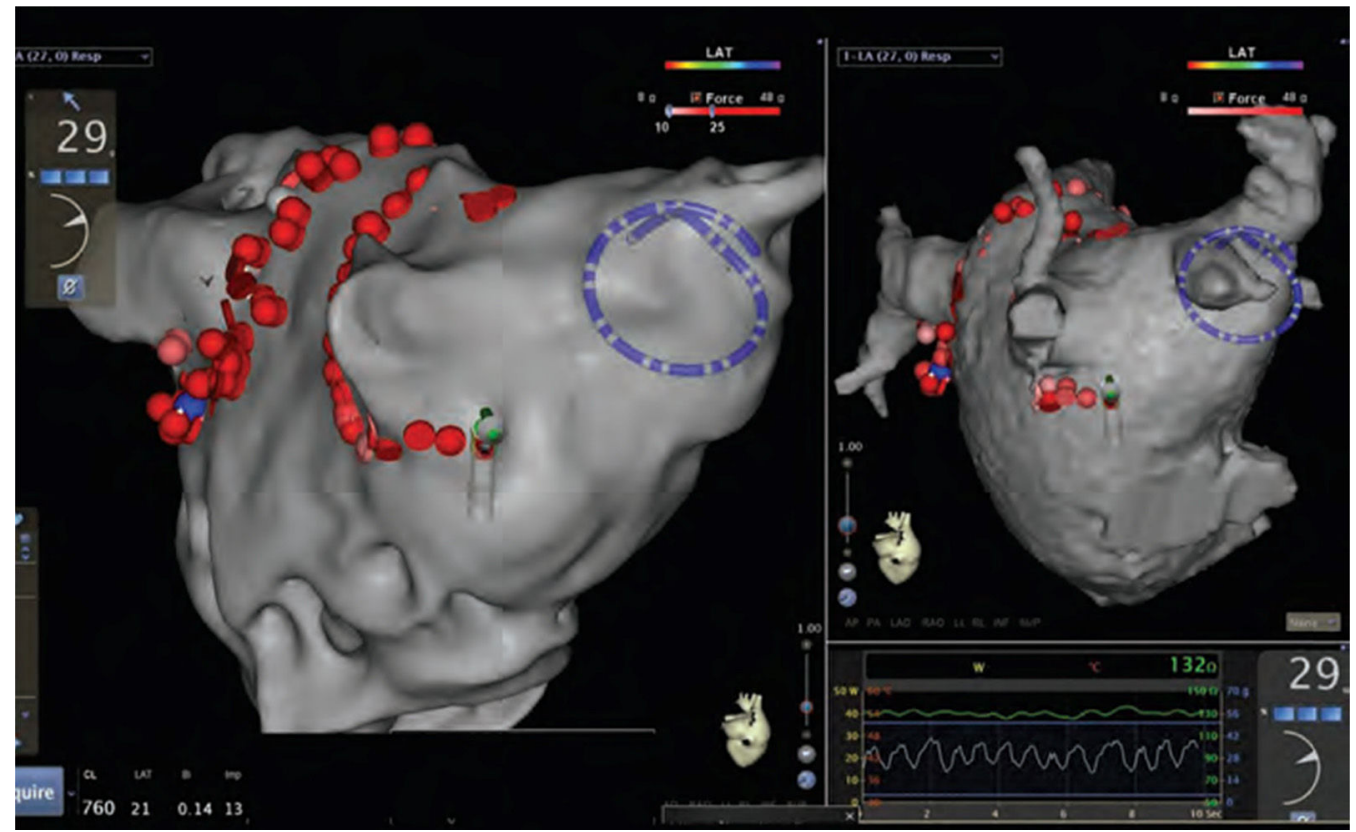

Figura 1 Imágenes de datos del catéter de fuerza de contacto Thermocool Smartouch durante aislamiento eléctrico de venas pulmonares. Panel izquierdo: mapa anatómico de la aurícula izquierda (vista posterior). El catéter de ablación de fuerza de contacto es visto durante la ablación punto por punto debajo de la vena pulmonar inferior derecha (VPID) con la correspondiente fuerza de contacto promedio de $29 \mathrm{~g}$ (panel izquierdo). Automáticamente, cada punto de ablación es codificado en color por el sistema en un rango desde rosado $(<10 \mathrm{~g})$ hasta rojo $(>25 \mathrm{~g})$, lo que muestra al operador datos en tiempo real de la fuerza de contacto entregada. El panel superior derecho muestra una tomografía computarizada de la aurícula izquierda en la misma orientación. El panel inferior derecho muestra trazos en tiempo real de la fuerza de contacto durante la administración de $10 \mathrm{~s}$ de lesiones de radiofrecuencia. La impedancia durante la entrega de lesiones se muestra en el trazo verde (Modificada de: Hoffmayer K, Gerstenfeld E. Contact force-sensing catheters. Curr Opin Cardiol 2015, 30:74-80).

fuerza de contacto cada $25,6 \mathrm{~ms}$. La fuerza de contacto se presenta de ambas formas, continuamente y como un valor promedio cada segundo sobre un sistema de mapeo electroanatómico (CARTO, Biosense Webster Inc., Diamond Bar, California, USA) (fig. 1).

El catéter de fuerza de contacto TactiCath utiliza un sensor de fuerza que es incorporado en la parte distal del catéter de ablación entre el segundo y el tercer electrodo. El sensor de este último consiste en un cuerpo deformable y tres fibras ópticas para cuantificar las deformaciones que se correlacionan con la fuerza aplicada a la punta del catéter. Por tanto, la aplicación de fuerza de contacto a la punta del catéter produce microdeformación de su cuerpo, ya sea estirándolo o comprimiéndolo, lo que provoca el cambio en la longitud de onda de la luz reflejada, que a su vez es proporcional a la fuerza de contacto aplicada a la punta. El sensor de fuerza de contacto tiene una sensibilidad aproximada de $1 \mathrm{~g}$. Las fuerzas individuales y la fuerza resultante se calculan cada 100 ms y se presentan de manera continua ${ }^{3}$ (fig. 2).

\section{Importancia de la fuerza de contacto}

Datos experimentales han demostrado que la presión de contacto en el tejido es el mayor determinante del tamaño de la lesión independiente de la irrigación de la punta del catéter ${ }^{4,5}$.
En el pasado se utilizaron algunos marcadores indirectos de contacto como la impedancia basal y su caída durante la ablación, la temperatura de la punta del catéter (para ablación no irrigada) y la atenuación de los electrogramas durante la ablación. La impedancia inicial podría esperar en correlacionarse con la fuerza de contacto de la punta del catéter; una fuerza de contacto alta podría incrementar la impedancia inicial al aumentar la interface tejido-electrodo, por acrecentar el área de interface electrodo tejido y reducir la de interface electrodo sangre.

El calentamiento del tejido durante radiofrecuencia podría traducirse en una caída de la impedancia tisular mientras que el cambio de impedancia refleja el tamaño de la lesión e indirectamente la fuerza de contacto ${ }^{6}$. Yokoyama et al. ${ }^{2}$ fueron los primeros en describir la relación existente entre fuerza de contacto electrodo tejido y tamaño de la lesión. A un poder y tiempo de aplicación constante, el incremento de la fuerza de contacto (2-40 g) aumenta significativamente la temperatura del tejido (a profundidad de 3-7 mm), así como la profundidad, el diámetro y el volumen de la lesión de radiofrecuencia. El tamaño de la lesión dependió más de la fuerza de contracto que del poder aplicado, encontrando lesiones más grandes con bajo poder $(30 \mathrm{~W})$ y fuerza de contacto mayor (30-40 g), comparados con lesiones generadas con mayor poder $(50 \mathrm{~W})$ y baja fuerza de contacto (2-10 g). Sin embargo, existe mayor posibilidad de generar explosión de gas y formación de trombos 


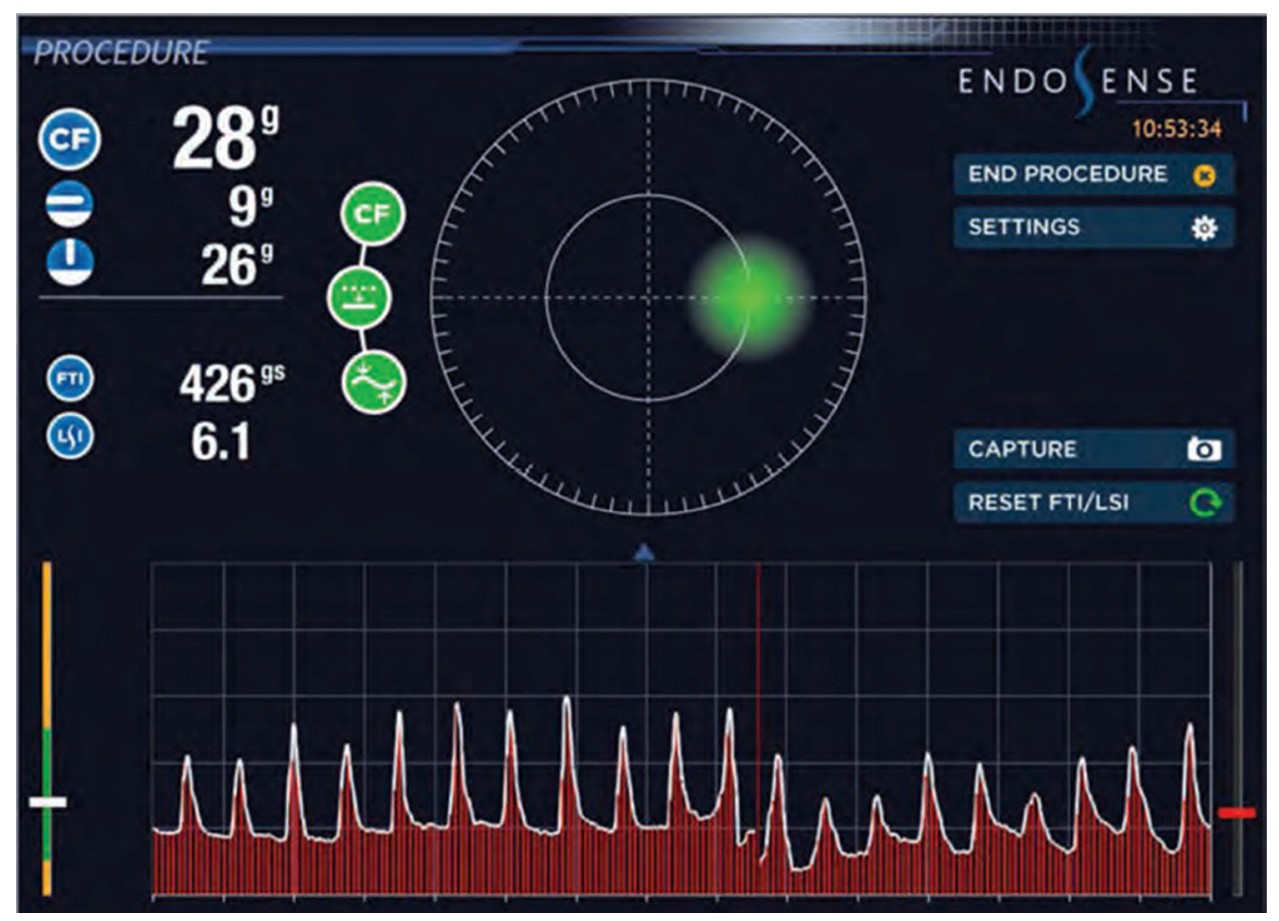

Figura 2 Captura de pantalla del módulo de fuerza de contacto EnSite. La parte superior izquierda de la pantalla muestra la fuerza de contacto en tiempo real en gramos y siguiendo directamente debajo de él la fuerza de contacto del vector horizontal y vertical, el intervalo fuerza tiempo ( $\mathrm{FTI}, \mathrm{g} / \mathrm{s}$ ) y el índice lesión-tamaño ( $\mathrm{LSI}, \mathrm{s} / \mathrm{g} / \mathrm{W})$. La parte central de la cuadrícula muestra la orientación espacial para la fuerza de contacto, mientras el punto verde representa la localización de la fuerza de contacto ejercida en la punta del catéter. La mitad inferior de la pantalla muestra la escala FTI con la fuerza total en el eje Y, totales la fuerza en el eje Y vs. el tiempo en el eje x (Modificada de: Hoffmayer K, Gerstenfeld E. Contact force-sensing catheters. Curr Opin Cardiol. 2015,30:74-80).

con mayor fuerza de contacto aplicada. En efecto, los trombos solo se presentaron con fuerzas de contacto mayores de $20 \mathrm{~g}$ y siempre se observaron en la parte proximal del borde del electrodo, en la interface tejido electrodo.

Es de notar también que la fuerza de contacto no siempre se correlaciona con la impedancia inicial o la disminución de la impedancia durante la ablación por radiofrecuencia. Existe una tendencia de impedancia mayor inicial con mayor fuerza de contacto, pero no es significativa.

La importancia reconocida de la fuerza de contacto es generada por la guía para el uso temprano de vainas deflectables para mantener mejor fuerza de contacto durante la ablación de la fibrilación atrial en algunos centros, y ahora con el advenimiento de los catéteres de fuerza de contacto ${ }^{7}$.

\section{Ablación de fibrilación atrial con el uso de la fuerza de contacto}

Desde el primer informe hecho por Haissaguerre et al. ${ }^{8}$ sobre la iniciación espontánea de fibrilación auricular por latidos ectópicos originados en las venas pulmonares ya hace más de 15 años, el campo de la ablación de la fibrilación auricular mediante radiofrecuencia, ha crecido de manera exponencial. La ablación con catéter se ha centrado en la creación de áreas de lesiones antrales circunferenciales alrededor de las venas pulmonares para crear aislamiento eléctrico ${ }^{9-11}$. Sin embargo, a pesar de lograr dicho aislamiento durante el procedimiento, la recurrencia de fibrilación auricular después de la ablación no es rara ${ }^{12}$ y casi siempre se asocia a la reconexión de la vena pulmonar ${ }^{13}$. La aplicación insuficiente de la fuerza de contacto durante la formación inicial de las lesiones, puede conducir a edema y lesiones no transmurales ineficaces que permiten la reconexión subaguda de la vena pulmonar cuando el edema se resuelve ${ }^{3}$. El objetivo es crear lesiones contiguas con suficiente transmuralidad, pero esto no es una tarea sencilla. La energía suministrada debe ser suficiente para generar la transmuralidad, pero no tan excesiva como para producir daños en las estructuras colaterales (esófago, arteria pulmonar y aorta) o daño directo en la aurícula (derrame pericárdico, taponamiento cardiaco).

Reddy et al. ${ }^{3}$ publicaron los resultados del primer ensayo multicéntrico [TOuCh + for catheter ablation (TOCCATA)] para evaluar el uso de catéteres de fuerza de contacto en el aislamiento de venas pulmonares ${ }^{3}$. Los autores estudiaron la relación entre los parámetros de fuerza de contacto durante el procedimiento y los resultados a los 12 meses, en 34 pacientes referidos para ablación de fibrilación auricular paroxística. El éxito era definido por cualquiera de los dos: no recurrencia de fibrilación atrial (ausencia de fibrilación auricular mayor a $30 \mathrm{~s}$ ) o recurrencia de fibrilación auricular con aislamiento de vena pulmonar (PVI) confirmado durante un segundo procedimiento de ablación.

El operador tenía acceso a la medición de la fuerza de contacto en tiempo real para guiar las aplicaciones. El éxito en el aislamiento eléctrico de las cuatro venas se alcanzó en 
el $100 \%$ de los pacientes. El análisis de la fuerza de contacto durante la ablación reveló 35\% de lesiones, con una fuerza de contacto baja $(<10 \mathrm{~g})$, y $52 \%$ de las lesiones fueron realizadas con fuerza de contacto entre 10 y $30 \mathrm{~g}$. A los 12 meses, todos los pacientes (100\%) tratados con una fuerza de contacto $<10 \mathrm{~g}$, tenían recurrencia, mientras que $80 \%$ de los pacientes tratados con una fuerza de contacto $>20 \mathrm{~g}$ estaban libres de fibrilación auricular $(p=0,01)$.

La recurrencia clínica también fue predecible con base en el número de lesiones con fuerza de contacto baja $(C F<5 g)$ : cuando se presentaron por lo menos dos lesiones con fuerza de contacto baja, la tasa de éxito a 12 meses fue del $40 \%$ en comparación con una tasa de éxito del $75 \%$ cuando se presentaron de 0 a 1 lesión con fuerza de contacto baja.

El ensayo EFFICAS I, reportado por Neuzil et al. ${ }^{14}$, fue un estudio multicéntrico prospectivo que evaluó la correlación entre la fuerza de contacto durante el procedimiento inicial y la presencia de gaps en las venas pulmonares medidos con un catéter de mapeo circular durante un estudio electrofisiológico a los tres meses. El objetivo fue comparar los sitios de reconexión de las venas pulmonares y evaluar los factores que pueden explicar lesiones no duraderas.

El procedimiento se realizó en 46 pacientes con fibrilación auricular paroxística. Durante este, el operador fue cegado a los parámetros de fuerza de contacto. La fuerza de contacto se evaluó por gramos de fuerza, así como por el intervalo de fuerza-tiempo ( $\mathrm{FTI}$, su sigla en inglés), una medida ampliamente aceptada de la fuerza de contacto definida como la fuerza de contacto integrada total sobre el momento de la entrega de energía de radiofrecuencia. En el seguimiento, 65\% de los pacientes mostraron al menos un gap en la circunferencia del aislamiento de la vena pulmonar. La ablación con fuerza de contacto baja y FTI menor de $400 \mathrm{~g} / \mathrm{s}$, mostró aumento de la probabilidad para la reconexión de la vena pulmonar $(p<0,001)$. Los sitios de reconexión se correlacionaron con lesiones con fuerza de contacto baja $(p<0,0001)$ y baja FTI $(p=0,0007)$. Se encontró que la fuerza de contacto y la FTI eran generalmente más altos en las venas pulmonares del lado derecho. Los autores concluyeron que la fuerza de contacto baja $(<10 \mathrm{~g})$ y los valores de la FTI (< $400 \mathrm{~g} / \mathrm{s}$ ) son fuertes predictores de la formación eventual de gap. Los parámetros óptimos recomendados por este estudio fueron una fuerza de contacto de $20 \mathrm{~g}$ y una FTI mínima de $400 \mathrm{~g} / \mathrm{s}$ para cada lesión entregada.

Por su parte, Sotomi et al. ${ }^{15}$ evaluaron el efecto de la fuerza de contacto en la reconexión aguda de la vena pulmonar en 57 pacientes sometidos a aislamiento de venas pulmonares. Se correlacionaron los segmentos reconectados con la fuerza de contacto. De un total de 4.421 lesiones de ablación, $285(6,4 \%)$ se asociaron con gaps presentes en la reconexión. Los valores promedio de la fuerza de contacto de los puntos con reconexión, fueron significativamente más bajos $(7,5 \pm 6,7$ vs. a 9,9 $\pm 8,4 \mathrm{~g}, \mathrm{p}<0,0001)$. Los autores encontraron que el valor óptimo de fuerza de contacto para evitar la reconexión aguda de la vena pulmonar con más del $95 \%$ de probabilidad, era de 10 a $22 \mathrm{~g}$, dependiendo de los segmentos de vena pulmonar individuales sometidos a ablación.

Así mismo, Park et al. ${ }^{16}$ evaluaron el efecto de la fuerza de contacto en la reconexión aguda de la vena pulmonar en 40 pacientes sometidos a aislamiento de venas pulmonares. Se necesitaron 1.926 lesiones para aislar 53 venas pulmonares. La reconexión aguda de la vena pulmonar se produjo en 35 venas pulmonares (23\%), la mayoría con la provocación de adenosina $(22 / 35,63 \%)$, el resto en forma espontánea $(13 / 35,37 \%)$. La fuerza de contacto era significativamente menor para las lesiones con segmentos de reconexión de $6 \mathrm{~mm}$ en comparación con regiones sin reconexión (media fuerza de contacto 5 frente a $11 \mathrm{~g}$; $\mathrm{p}<0,0001$; FTI 225 frente a $415 \mathrm{~g} ; \mathrm{p}<0,0001)$. La mayoría de las reconexiones $(86 \%)$ ocurrieron en los sitios en los que las lesiones fueron entregadas con una fuerza de contacto media menor de $10 \mathrm{~g}$ y FTI menor de $400 \mathrm{~g} / \mathrm{s}$; el restante $14 \%$ ocurrió en los sitios de ablación con una distancia interlesión prolongada (> $5 \mathrm{~mm}$ ).

Ullah et al. ${ }^{17}$ hicieron un análisis detallado de la relación entre los cambios de impedancia, amplitud de electrogramas y fuerza de contacto en 15 pacientes sometidos a aislamiento de venas pulmonares en fibrilación auricular persistente. Su objetivo era establecer parámetros adecuados para ablación por radiofrecuencia e identificar un punto de meseta para la ablación. La mayoría de los pacientes $(11 / 15,73 \%)$ fueron sometidos a ablación con navegación robótica a distancia (Sensei Robotic sistema de catéter, Hansen Medical, Inc., California, EE.UU.). Se midió la fuerza de contacto utilizando la FTI (g/s). La FTI durante la ablación se correlacionó con la caída de la impedancia (Spearman $\mathrm{p}$ $0,79 ; \mathrm{p}<0,0005)$, con una meseta en $500 \mathrm{~g} / \mathrm{s}$, más allá de la cual no se encontró beneficio adicional de una mayor ablación. Una aplicación menor de 10 segundos tuvo una mayor variación en la caída de la impedancia, mientras aplicaciones de más mas de 10 segundos fueron más consistentes y se correlacionaron con la FTI. Los autores concluyeron que la caída de impedancia $(7,5 \%)$ puede ser un sustituto más preciso de la formación de lesión que la atenuación de los electrogramas, y en forma más significativa, el objetivo debe ser una FTI de $500 \mathrm{~g} / \mathrm{s}$ con una duración mínima de ablación de $10 \mathrm{~s}$.

También se ha demostrado que el empleo de catéteres de fuerza de contacto reduce el tiempo del procedimiento requerido para el aislamiento de venas pulmonares. Kimura et al. ${ }^{18}$ aleatorizaron 38 pacientes consecutivos $(28 / 38$ paroxística) sometidos a aislamiento de venas pulmonares guiados por fuerza de contacto frente a ablación sin fuerza de contacto. En el grupo sin fuerza de contacto, el operador era cegado a toda la fuerza de contacto; en el grupo con fuerza de contacto, la fuerza de contacto se mantuvo entre 10 y $20 \mathrm{~g}$. El aislamiento eléctrico fue logrado en forma exitosa en ambos grupos, con un promedio mayor de fuerza de contacto en el grupo guiado por catéteres de fuerza de contacto, tanto en las venas izquierdas como en las derechas $(11,11 \pm 4,3$ frente a $5,9 \pm 4,5 \mathrm{~g}, \mathrm{p}<0,001)$ para las venas izquierdas y $12,1 \pm 4,8$ frente a $9,8 \pm 6,6 \mathrm{~g}, \mathrm{p}<0,001$, por las venas del lado derecho. Hubo una reducción significativa de los tiempos de procedimiento con el uso de fuerza de contacto: $59 \pm 16 \mathrm{~min}$ frente a $96 \pm 39 \min (p<0,001)$. El grupo de ablación guiada por fuerza de contacto también tenía una reducción significativa en el número de gaps residuales de conducción que requirieron retoques de ablación para completar ablación circunferencial $(2,8 \pm 1,9$ frente a $6,3 \pm$ en 3,$0 ; p<0,001$ ). Luego de seis meses de seguimiento, el $94,7 \%$ de los pacientes en el grupo guiado por fuerza de contacto frente al $84 \%$ sin ésta, estaban libres de cualquier arritmia auricular $(p=0,34)$. Los autores concluyeron que 
el empleo de catéter con medición de fuerza de contacto para el aislamiento circunferencial de venas pulmonares, redujo el tiempo del procedimiento y el número de lesiones adicionales de ablación, aunque no hubo diferencia estadísticamente significativa en la tasa de éxito.

Natale et al. ${ }^{19}$ llevaron a cabo un estudio prospectivo, multicéntrico, no aleatorizado (SMART AF), en el que incluyeron 172 pacientes, con el objetivo de evaluar la seguridad y efectividad de la ablación de fibrilación auricular con el uso de catéter de fuerza de contacto. Los investigadores reportaron que cuando la fuerza de contacto se mantuvo por más del $80 \%$ del tiempo en los rangos propuestos $(17,9 \pm 9,4 \mathrm{~g})$, el éxito fue 4,5 veces más probable ( $p=0,0054$; IC 95\%; 1,53 a 11,79).

Reddy et al. ${ }^{20}$ publicaron el ensayo TOCCASTAR, en el cual se evaluó la seguridad y la eficacia del catéter de fuerza de contacto en la ablación de fibrilación auricular paroxística. Se incluyeron 300 pacientes, fue un estudio controlado, prospectivo, aleatorizado, multicéntrico. El punto primario de efectividad fue el aislamiento eléctrico de todas las venas pulmonares y la ausencia de fibrilación auricular sintomática a los 12 meses. El punto primario de seguridad fue la ausencia de eventos adversos serios. El análisis fue elaborado con el propósito de establecer no inferioridad. Todas las venas pulmonares fueron aisladas en ambos grupos. La efectividad se logró en $67,8 \%$ de los pacientes con fuerza de contacto, frente al 69,4\% del grupo control (diferencia absoluta, -1,6\%; IC 95\%, -10,7\%; $p=0,0073$ para la no inferioridad). El punto primario de seguridad ocurrió en 1,97\% y el $1,40 \%$ de los pacientes con fuerza de contacto frente al grupo control, respectivamente (diferencia absoluta, 0,57\%; IC 95\%, 3,61\%; $p=0,0004$ para la no inferioridad). Los autores concluyeron que la ablación de fibrilación con el empleo de catéteres de fuerza de contacto no es inferior al uso de catéteres convencionales en términos de efectividad y seguridad.

En fecha reciente se publicó el ensayo EFFICAS II, reportado por Kautzner et al., ${ }^{21}$ el cual es un estudio multicéntrico prospectivo para evaluar la ablación de fibrilación auricular guiada por catéteres con fuerza de contacto en la reducción de gaps. Se encontró una reducción significativa en la presencia de éstos; a los 3 meses de seguimiento el $85 \%$ de los pacientes permanecían con venas aisladas vs. el $72 \%$ del grupo control $(p=0,037)$. El EFFICAS ॥ fue el primer estudio que evaluó en forma prospectiva el uso de catéteres de fuerza de contacto en la ablación de fibrilación auricular, demostrando que la utilización de una fuerza de contacto mínima de $20 \mathrm{~g}$ y un FTI de $400 \mathrm{~g} / \mathrm{s}$, se traduce en una mayor proporción de venas aisladas a los tres meses de seguimiento.

En un reciente metaanálisis publicado por Afzal et al. ${ }^{22}$ se incluyeron 9 estudios (1.148 pacientes), teniendo en cuenta como punto primario el riesgo relativo de recurrencia de fibrilación auricular a los 12 meses, y como puntos secundarios el tiempo total del procedimiento, la exposición a fluoroscopia, y la duración de aplicación de radiofrecuencia. En comparación con la tecnología estándar, el empleo de catéteres de fuerza de contacto demostró una reducción del $37 \%$ en el riesgo relativo de recurrencia, con una media de seguimiento de 12 meses (riesgo relativo 0,63; IC 95\% $0,44-0,91 ; p=0,01)$, con una reducción de 7,3 minutos en la duración de aplicación de radiofrecuencia (IC95\%-14,05 a
$-0,55 ; p=0,03)$. No hubo diferencia en la duración total del procedimiento ni en el tiempo de fluoroscopia.

En el metaanálisis de Shurrab et al. ${ }^{23}$ se incluyeron 11 estudios (2 estudios clínicos y 9 cohortes), con un total de 1.428 pacientes. El seguimiento promedio fue entre $10 \mathrm{a}$ 53 semanas. La tasa de recurrencia fue menor en el grupo de la fuerza de contacto $(35,1 \%$ frente a $45,5 \%$, OR 0,62 ; IC 95\% 0,45-0,86, $\mathrm{p}=0,004)$. La duración total del procedimiento y el tiempo de fluoroscopia fueron más cortos en el grupo de fuerza de contacto (tiempo de procedimiento: 156 frente a 173 minutos, promedio de diferencia estandarizada IC 0,85 [95\% -1,48 a-0,2], $p=0,009$; tiempo de fluoroscopia: 28 frente a 36 minutos, diferencia de medias estandarizada IC 0,94 [95\%-1,66-0,21], p=0,01). La tasa de complicaciones mayores fue menor en el grupo de fuerza de contacto, pero no estadísticamente significativa $(1,3 \%$ frente a 1,9\%, OR 0,71 [95\% IC 0,29 a 1,73], $p=0,45)$.

\section{Recomendaciones}

Con base en la evidencia actual se recomienda el uso de catéteres con sensor de fuerza de contacto en la ablación de fibrilación auricular paroxística (recomendación clase IIA, nivel de evidencia $B$ ).

\section{Conclusiones}

Gran parte del conocimiento actual que se tiene en cuanto a la utilidad de la medición continua de fuerza de contacto durante procedimientos de ablación por radiofrecuencia, proviene del aislamiento de venas pulmonares para el tratamiento de la fibrilación auricular.

Durante el procedimiento de aislamiento de venas pulmonares, el objetivo de fuerza de contacto debe ser por lo menos $10 \mathrm{~g}$ con una meta de $20 \mathrm{~g}$, en tanto que el FTI debe ser por lo menos de $400 \mathrm{~g} / \mathrm{s}$, con una meta de $500 \mathrm{~g} / \mathrm{s}$. Las lesiones deben realizarse con una duración mínimo de $10 \mathrm{~s}$.

Las fuerzas de contacto bajas menores a $10 \mathrm{~g}$ o a $400 \mathrm{~g} / \mathrm{s}$ de $\mathrm{FTI}$, se han correlacionado en la mayoría de los estudios con mayor incidencia de reconexión aguda y crónica, de modo que se deben evitar.

Por esto el uso de catéteres de fuerza de contacto durante el aislamiento de venas pulmonares se correlaciona con mejores resultados clínicos a un año, menores gaps en las líneas de ablación a tres meses y menor tiempo de procedimiento, por lo que a pesar de que la evidencia actual está en recolección, promete ser una herramienta de gran utilidad para mejorar los resultados finales en la ablación de la fibrilación auricular.

\section{Conflicto de intereses}

Los autores declaran no tener conflicto de intereses.

\section{Bibliografía}

1. Shah $\mathrm{DC}$, Lambert $\mathrm{H}$, Nakagawa $\mathrm{H}$, et al. Area under the real-time contact force curve (force-time integral) predicts radiofrequency lesion size in an in vitro contractile model. J Cardiovasc Electrophysiol. 2010;21:1038-43. 
2. Yokoyama K, Nakagawa H, Shah DC, et al. Novel contact force sensor incorporated in irrigated radiofrequency ablation catheter predicts lesion size and incidence of steam pop and thrombus. Circ Arrhythm Electrophysiol. 2008;1:354-62.

3. Reddy VY, Shah D, Kautzner J, et al. The relationship between contact force and clinical outcome during radiofrequency catheter ablation of atrial fibrillation in the TOCCATA study. Heart Rhythm. 2012;9:1789-95.

4. Zheng X, Walcott GP, Hall JA, et al. Electrode impedance: an indicator of electrode-tissue contact and lesion dimensions during linear ablation. J Interv. Card Electrophysiol. 2000;4:645-54.

5. Strickberger SA, Vorperian VR, Man KC, et al. Relation between impedance and endocardial contact during radiofrequency catheter ablation. Am Heart J. 1994;128:226-9.

6. Avitall B, Mughal K, Hare J, et al. The effects of electrodetissue contact on radiofrequency lesion generation. Pacing Clin Electrophysiol. 1997;20:2899-910.

7. Gerstenfeld EP. Contact force-sensing catheters: evolution or revolution in catheter ablation technology? Circ Arrhythm Electrophysiol. 2014;7:5-6.

8. Haissaguerre M, Jais P, Shah DC, et al. Spontaneous initiation of atrial fibrillation by ectopic beats originating in the pulmonary veins. N Engl J. Med. 1998;339:659-66.

9. Oral H, Pappone C, Chugh A, et al. Circumferential pulmonaryvein ablation for chronic atrial fibrillation. $N$ Engl J Med. 2006;354:934-41.

10. Pappone C, Rosanio S, Augello G, et al. Mortality, morbidity, and quality of life after circumferential pulmonary vein ablation for atrial fibrillation: outcomes from a controlled nonrandomized long-term study. J Am Coll Cardiol. 2003;42:185-97.

11. Dewire J, Calkins $H$. Update on atrial fibrillation catheter ablation technologies and techniques. Nat Rev Cardiol. 2013;10:599-612.

12. January CT, Wann LS, Alpert JS, et al. 2014 AHA/ACC/HRS Guideline for the management of patients with atrial fibrillation. J Am Coll Cardiol. 2014;64:e1-76.

13. Gerstenfeld EP, Callans DJ, Dixit S, et al. Incidence and location of focal atrial fibrillation triggers in patients undergoing repeat pulmonary vein isolation: implications for ablation strategies. J Cardiovasc Electrophysiol. 2003;14:685-90.
14. Neuzil P, Reddy VY, Kautzner J, et al. Electrical reconnection after pulmonary vein isolation is contingent on contact force during initial treatment: results from the EFFICAS I study. Circ Arrhythm Electrophysiol. 2013;6:327-33.

15. Sotomi $\mathrm{Y}$, Kikkawa $\mathrm{T}$, Inoue $\mathrm{K}$, et al. Regional difference of optimal contact force to prevent acute pulmonary vein reconnection during radiofrequency catheter ablation for atrial fibrillation. J Cardiovasc Electrophysiol. 2014;25: 941-7.

16. Park $\mathrm{Cl}$, Lehrmann $\mathrm{H}$, Keyl $\mathrm{C}$, et al. Mechanisms of pulmonary vein reconnection after radiofrequency ablation of atrial fibrillation: the deterministic role of contact force and interlesion distance. J Cardiovasc Electrophysiol. 2014;25: 701-8.

17. Ullah W, Hunter RJ, Baker V, et al. Target indices for clinical ablation in atrial fibrillation: insights from contact force, electrogram, and biophysical parameter analysis. Circ Arrhythm Electrophysiol. 2014;7:63-8.

18. Kimura M, Sasaki S, Owada S, et al. Comparison of lesion formation between contact force-guided and nonguided circumferential pulmonary vein isolation: a prospective, randomized study. Heart Rhythm. 2014;11:984-91.

19. Natale A, Reddy VY, Monir G, et al., Paroxysmal AF. Catheter Ablation With a Contact Force Sensing Catheter Results of the Prospective, Multicenter SMART-AF Trial. J Am Coll Cardiol. 2014;64:647-56.

20. Reddy VY, Dukkipati SR, Neuzil P, et al. Randomized, controlled trial of the safety and effectiveness of a contact force-sensing irrigated catheter for ablation of paroxysmal atrial fibrillation. Circulation. 2015;132:907-15.

21. Kautzner J, Neuzil P, Lambert H, et al. EFFICAS II: optimization of catheter contact force improves outcome of pulmonary vein isolation for paroxysmal atrial fibrillation. Europace. 2015;17:1229-35.

22. Afzal MR, Chatta J, Samanta A, et al. Use of contact force sensing technology during radiofrequency ablation reduces recurrence of atrial fibrillation: A systematic review and meta analysis. Heart Rhythm. 2015;12:1990-6.

23. Shurrab M, Di Biase L, Biceno D, et al. Impact of contact force technology on atrial fibrillation ablation: a meta-analysis. J Am Heart Assoc. 2015;31:S222. 